\section{Commentary: Aortic wrapping remains an incomplete solution for treatment of acute type $A$ aortic dissections}

\author{
Christopher Lau, MD, and Leonard N. Girardi, MD
}

"Sooner or later, everything old is new again" applies as much to cardiac surgery as it does to popular culture, literature, and fashion. In this issue of the Journal, Vento and colleagues ${ }^{1}$ attempt to reinvigorate interest in the technique of aortic wrapping. Although this concept has been discussed since the 1940s, before the advent of cardiopulmonary bypass, it has never garnered widespread use in aneurysm repair. Problems with erosion, rupture, wrap migration, and pseudoaneurysms limited the adoption of aortic wrapping. $^{2-4}$ In addition, elective repair of ascending aneurysms carries a low operative risk and has excellent long-term durability, negating the need for a lesser procedure. ${ }^{5}$ In this study, the technique was applied to patients with acute type A aortic dissection (ATAAD) deemed high-risk for aortic replacement. Although the group achieved a commendable operative mortality of $9 \%$ for ATAAD compared with the relatively higher mortality rates seen in large databases, such as the International Registry of Aortic Dissection, ${ }^{6}$ the result is achieved with a substantial number of complications and need for early reinterventions.

The limitations of aortic wrapping stem from the ability of the procedure to treat only one aspect of the deadly trio in ATAAD. Although the wrap may help prevent aortic rupture, it does nothing to prevent progression of the dissection, acute aortic insufficiency, or end-organ malperfusion. A staggering $31 \%$ of patients required reinterventions on

\footnotetext{
From the Department of Cardiothoracic Surgery, Weill Cornell Medicine, New York, NY.

Disclosures: The authors reported no conflicts of interest.

The Journal policy requires editors and reviewers to disclose conflicts of interest and to decline handling or reviewing manuscripts for which they may have a conflict of interest. The editors and reviewers of this article have no conflicts of interest.

Received for publication Nov 5, 2020; revisions received Nov 5, 2020; accepted for publication Nov 8, 2020; available ahead of print Nov 20, 2020.

Address for reprints: Leonard N. Girardi, MD, Department of Cardiothoracic Surgery, Weill Cornell Medicine, 525 East 68th St, M-404, New York, NY 10065 (E-mail: lngirard@med.cornell.edu).

J Thorac Cardiovasc Surg 2022;164:1421-2

$0022-5223 / \$ 36.00$

Copyright (c) 2020 by The American Association for Thoracic Surgery

https://doi.org/10.1016/j.jtcvs.2020.11.027
}



CENTRAL MESSAGE

Aortic wrapping may avert

rupture of an aortic dissection but does not prevent malperfusion or aortic insufficiency. Acceptable outcomes may be possible with vigilant postoperative monitoring.

the same admission for these complications, including ascending aortic stents for progression of the dissection flap, transcatheter aortic valve replacement for acute aortic insufficiency, and numerous vascular procedures for new malperfusion syndromes. One of the principle goals of ATAAD surgery is to resect the primary tear, thereby improving true lumen perfusion, which readily treats existing malperfusion syndromes and prevents new malperfusion from developing. Aortic wrapping clearly does not achieve this goal. Interestingly, wrapping of nondissected aneurysms does not prevent the occurrence of new aortic dissection. ${ }^{7}$ Although aortic remodeling has been seen in the wrapped portion of the aorta, data on long-term durability remain sparse.

Aortic wrapping may have its place in the management of ATAAD, and the authors admit that it is considered for only high-risk patients. However, the criteria for defining "high-risk" are unclear. Patients at risk with any cardiopulmonary bypass, such as those with severely compromised pulmonary function, are clearly at high risk for aortic replacement, but other criteria used by the authors, such as diabetes and peripheral artery disease, are somewhat questionable. A significant proportion of the patients were actually quite young (as young as 46 years), and it is unclear what comorbidities in this normally low-risk population precluded cardiopulmonary bypass. 
Furthermore, the acceptable aortic characteristics were quite stringent and excluded any patients with malperfusion or aortic insufficiency, meaning that no dissection involved the aortic root. This subset is at lower risk in terms of aortic characteristics, and it is not surprising that good operative outcomes are achievable with stringent patient selection. Perhaps with stringent selection, even medical management can achieve good outcomes for a specific, well-defined subset of patients, including those chosen for aortic wrapping in this study. In the International Registry of Aortic Dissection database, medical management in an unselected group had a mortality of only $57 \%$, so nearly one-half of those managed without surgery survived. ${ }^{6}$

Aortic wrapping is used by a limited number of surgeons who have experience in the technique and only for a specific subset of patients presenting with ATAAD. Although short- and mid-term outcomes are acceptable, careful monitoring is required to manage early complications, and long-term durability remains in question. The ideal population for aortic wrapping needs further clarification.

\section{References}

1. Vento V, Multon S, Ramadan R, Deleuze P, Fabre D, Guihaire J, et al. Outcomes of urgent aortic wrapping for acute type A aortic dissections. J Thorac Cardiovasc Surg. 2022;164:1412-20.

2. Bauer M, Grauhan O, Hetzer R. Dislocated wrap after previous reduction aortoplasty causes erosion of the ascending aorta. Ann Thorac Surg. 2003;75: 583-4.

3. Dhillon JS, Randhawa GK, Straehley CJ, McNamara JJ. Late rupture after Dacron wrapping of aortic aneurysms. Circulation. 1986;74(3 Pt 2):I11-4.

4. Neri E, Massetti M, Tanganelli P, Capannini G, Carone E, Tripodi A, et al. Is it only a mechanical matter? Histologic modifications of the aorta underlying external banding. J Thorac Cardiovasc Surg. 1999;118:1116-8.

5. Gaudino M, Lau C, Munjal M, Avgerinos D, Girardi LN. Contemporary outcomes of surgery for aortic root aneurysms: a propensity-matched comparison of valvesparing and composite valve graft replacement. J Thorac Cardiovasc Surg. 2015; 150:1120-9.e1.

6. Evangelista A, Isselbacher EM, Bossone E, Gleason TG, Di Eusanio M, Sechtem U, et al. Insights from the international registry of acute aortic dissection: a 20-year experience of collaborative clinical research. Circulation. 2018;137: 1846-60.

7. Plonek T. A meta analysis and systematic review of wrapping of the ascending aorta. J Card Surg. 2014;29:809-15.

\title{
Commentary: Urgent aortic wrapping for acute type $A$ aortic dissection: New hat for an old trick?
}

\author{
Nicholas T. Kouchoukos, MD
}

Wrapping of the ascending aorta as a method of treatment for aneurysmal dilatation was first reported by Bahnson and Nelson in 1956. ${ }^{1}$ After partially excising a large ascending aortic aneurysm, they used a Scultetus binder

\footnotetext{
From the Division of Cardiovascular and Thoracic Surgery, Missouri Baptist Medical Center, BJC Healthcare, St. Louis, Mo.

Disclosures: The author reported no conflicts of interest.

The Journal policy requires editors and reviewers to disclose conflicts of interest and to decline handling or reviewing manuscripts for which they may have a conflict of interest. The editors and reviewers of this article have no conflicts of interest.

Received for publication Nov 5, 2020; revisions received Nov 5, 2020; accepted for publication Nov 8, 2020; available ahead of print Nov 19, 2020.

Address for reprints: Nicholas T. Kouchoukos, MD, Missouri Baptist Medical Center, 3023 N Ballas Rd, Suite 150D, St Louis, MO 63131 (E-mail: ntkouch@aol.com). J Thorac Cardiovasc Surg 2022;164:1422-3

$0022-5223 / \$ 36.00$

Copyright (c) 2020 by The American Association for Thoracic Surgery

https://doi.org/10.1016/j.jtcvs.2020.11.025
}

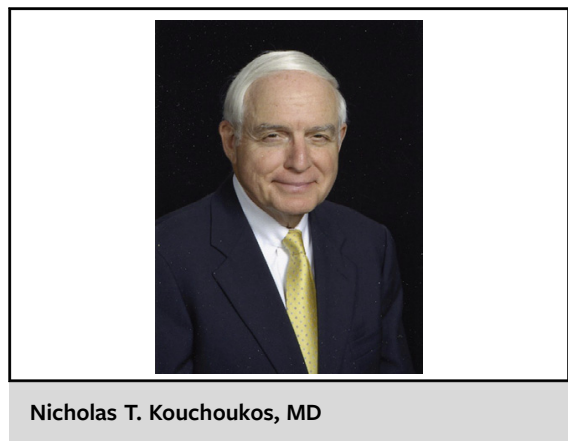

CENTRAL MESSAGE

Aortic wrapping may be a suitable option for treatment of acute type $\mathrm{A}$ aortic dissection in elderly and high-risk patients, but more patients and longer followup are needed.

to encircle the ascending aorta. Two of the 4 patients in whom the technique was used had chronic type A aortic dissections. Since that time, wrapping of the ascending aorta, with or without concomitant partial excision of the aortic 